\title{
Efficient evaluation of shortest travel-time path queries through spatial mashups
}

\author{
Detian Zhang' ${ }^{1}$ Chi-Yin Chow ${ }^{2}$ An Liu ${ }^{3,4}$. \\ Xiangliang Zhang ${ }^{3}$ Q Qingzhu Ding ${ }^{4}$ Qing $\mathrm{Li}^{2,5}$
}

Received: 1 October 2016 / Revised: 3 December 2016 / Accepted: 19 December 2016/

Published online: 7 January 2017

(C) Springer Science+Business Media New York 2017

\begin{abstract}
In the real world, the route/path with the shortest travel time in a road network is more meaningful than that with the shortest network distance for location-based services (LBS). However, not every LBS provider has adequate resources to compute/estimate travel time for routes by themselves. A cost-effective way for LBS providers to estimate travel time for routes is to issue external route requests to Web mapping services (e.g., Google Maps, Bing Maps, and MapQuest Maps). Due to the high cost of processing such external route requests and the usage limits of Web mapping services, we take the advantage of
\end{abstract}

An Liu

an.liu@kaust.edu.sa

Detian Zhang

detian.cs@gmail.com

Chi-Yin Chow

chiychow@cityu.edu.hk

Xiangliang Zhang

xiangliang.zhang@kaust.edu.sa

Qingzhu Ding

dqz48548263@qq.com

Qing Li

itqli@cityu.edu.hk

1 School of Digital Media, Jiangnan University, Wuxi, China

2 Department of Computer Science, City University of Hong Kong, Kowloon, Hong Kong

3 King Abdullah University of Science and Technology, Thuwal 23955-6900, Kingdom of Saudi Arabia

4 School of Computer Science, Soochow University, Suzhou, China

5 Multimedia Software Engineering Research Center, City University of Hong Kong Shenzhen Research Institute, Shenzhen, Guangdong, China 
direction sharing, parallel requesting and waypoints supported by Web mapping services to reduce the number of external route requests and the query response time for shortest travel-time route queries in this paper. We first give the definition of sharing ability to reflect the possibility of sharing the direction information of a route with others, and find out the queries that their query routes are independent with each other for parallel processing. Then, we model the problem of selecting the optimal waypoints for an external route request as finding the longest simple path in a weighted complete digraph. As it is a MAX SNP-hard problem, we propose a greedy algorithm with performance guarantee to find the best set of waypoints in an external route request. We evaluate the performance of our approach using a real Web mapping service, a real road network, real and synthetic data sets. Experimental results show the efficiency, scalability, and applicability of our approach.

Keywords Path queries · Travel time - Spatial mashups · Direction sharing ·

Parallel requesting $\cdot$ Waypoints $\cdot$ Web mapping services

\section{Introduction}

A spatial (or GIS/mapping) mashup [16, 35-38] provides a cost-effective way for a Web or mobile application that combines data, representation, and/or functionality from at least one Web mapping service and other local/external services to create a new application. It becomes more and more popular along with the development of Web mapping services and the ubiquity of Internet access and GPS-enabled mobile devices. Typical Web mapping services are Google Maps [8], MapQuest Maps [22] and Microsoft Bing Maps [23]. Based on the latest statistics of Programmable Web [25], the spatial mashup is the most popular one among all types of mashups including search mashups, social mashups, etc.

In the real world, the route with the shortest travel time (e.g., driving, walking and cycling time) in a road network is more meaningful than the route with the shortest network distance for location-based services (LBS) (see Fig. 1). Since travel time is highly dynamic due to many realistic factors, e.g., heterogeneous traffic conditions and traffic accidents, it is difficult for an LBS provider to perform travel route estimation/computation because of huge deployment cost. However, it is not a big problem for Web mapping service providers, as they have adequate resources to collect data from historical traffic statistics and/or continuously monitor the real-time traffic in road networks; besides, most of existing Web mapping services provide user-friendly APIs for applications to access travel route information, e.g., the Google Maps Directions API [27] and the MapQuest Directions Web Service [21].

Therefore, a typical and popular application scenario using spatial mashups is that an LBS provider subscribes the travel route information based on live traffic conditions from Web mapping services through their APIs to answer location-based queries for its own users [16, 35-38]. For example, Yelp.com [34], which is a leading Internet consumer rating and review Web site, outsources its mapping services to Google Maps [8], among which retrieving and then displaying the travel route information from a user's current/specified location to a point-of-interest (POI) makes the Web site more intuitive and attractive.

However, retrieving the travel route information through spatial mashups suffers from the following two critical limitations [36, 37]: (1) It is costly to access travel route information from a Web mapping service, e.g., retrieving travel time from the Microsoft MapPoint Web service to a database engine takes $502 \mathrm{~ms}$ while the time needed to read a cold and hot $8 \mathrm{~KB}$ buffer page from disk is $27 \mathrm{~ms}$ and $0.0047 \mathrm{~ms}$, respectively [14]. (2) There is a charge on the number of requests issued to a Web mapping service, e.g., the Google Maps Directions 


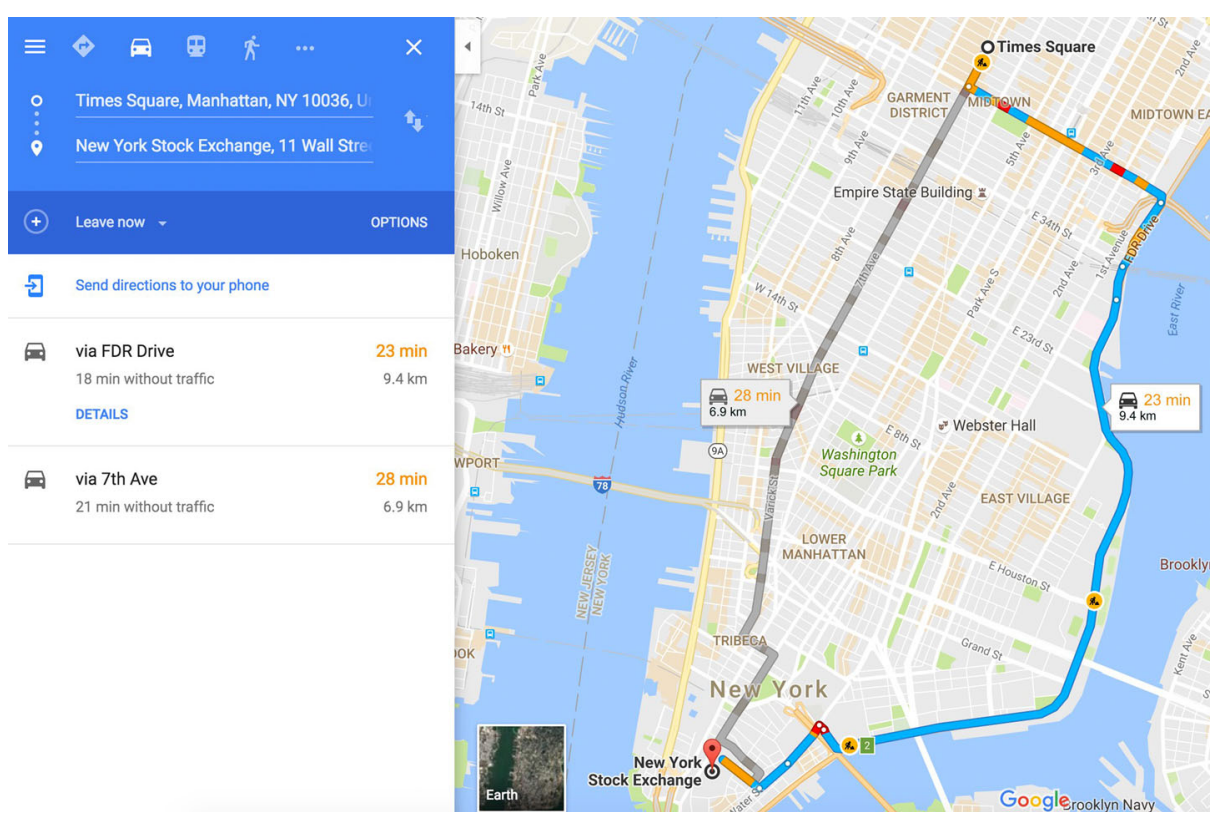

Fig. 1 The two different routes from Times Square to New York Stock Exchange by Google Maps (the blue/right one is with the shortest travel time, which is recommended by Google, and the gray/left one is with the shortest network distance)

API [27] allows only 2,500 requests per day for evaluation users and 100,000 requests per day for business license users [9]. An LBS provider needs to pay for higher usage limits. Therefore, when an LBS provider endures high workload, e.g., a large number of concurrent user queries, it needs to issue a large number of external route requests to Web mapping services, which not only result in high business operation cost, but also induce long response time to querying users.

In this paper, we aim to explore direction (or route/path) sharing optimization and appropriate waypoints in external route requests with parallel requesting to Web mapping services to reduce the number of external route requests and the response time to querying users for LBS providers.

Given user $u_{i}$ 's shortest travel-time path query $q_{i}=\left(o_{i}, d_{i}\right)$, the basic idea of the direction sharing optimization is that the route information from query origin $o_{i}$ to query destination $d_{i}$ (i.e., $R\left(o_{i} \rightarrow d_{i}\right)$ ) can be shared with another user $u_{j}$ 's query $q_{j}=\left(o_{j}, d_{j}\right)$ if both of its origin $o_{j}$ and destination $d_{j}$ are located in $R\left(o_{i} \rightarrow d_{i}\right)$. To reflect the opportunity of sharing the direction information of an external route request, we also formally define the sharing ability of a route. Besides, the queries whose querying routes are independent, i.e., they are not the sub-route of each other, are detected out for parallel processing to reduce the user query response time.

As most of existing Web mapping services that support adding waypoints into a route request, e.g., Google Maps allows up to eight intermediate waypoints in a route request for evaluation users and 23 intermediate waypoints for premier users [9], we attempt to select optimal waypoints in a route request to further reduce the number of external route requests and the query response time to users. We first model the problem of selecting optimal waypoints in a route request, i.e., the route through those waypoints can be shared 
by the largest number of queries, as the problem of finding the longest simple path in a weighted complete digraph. As it is a MAX SNP-hard problem, i.e., there is no polynomialtime algorithm that can even find a constant factor approximation for the problem unless $\mathrm{P}=\mathrm{NP}$ [13], we propose a greedy algorithm with performance guarantee to find the best set of waypoints in an external route request. The algorithm guarantees that at least $\left\lfloor\frac{m}{2}\right\rfloor$ queries in the query set $\mathcal{Q}$ can share the direction information of a returned route from Web mapping services, and tries to maximize the direction sharing optimization of the route at the same time, where $m$ is the number of waypoints in a route request.

In general, the main contributions of our work in this paper can be summarized as follows:

- We formally define the sharing ability of a route to reflect its direction sharing power, and design an algorithm to compute it.

- We find out the queries whose querying routes are independent for parallel processing to reduce the query response time to users without bringing in additional external route requests.

- We formulate the problem of selecting optimal waypoints in a route request, and propose a greedy algorithm with performance guarantee to solve it.

- We design an algorithm with all the optimizations (i.e., direction sharing, parallel requesting, and waypoint utilization) proposed in the paper.

- We conduct extensive experiments to evaluate the performance of the proposed optimizations using real a Web mapping service, a real road network, real and synthetic data sets.

The remainder of this paper is organized as follows. Section 2 highlights related work. Section 3 describes the system model. Section 4 presents the direction sharing optimization. Section 5 describes the route waypoint optimization. Section 6 gives an algorithm with all the optimizations proposed in the paper. Experimental results are analyzed in Section 7. Finally, Section 8 concludes this paper.

\section{Related work}

Queries have been widely used in our daily life [11, 15, 17-20, 32, 41], among which the shortest path query is one of the most important types. Existing shortest path query processing algorithms in road networks can be categorized into two main models:

(1) Time-independent road networks. In this model, shortest path query processing algorithms assume that the cost or weight of a road segment (e.g., distance or travel time) is constant (e.g., [26, 33, 39]). These algorithms mainly rely on pre-computed distance or travel time information of road segments in road networks. However, the actual travel time of a road segment may vary significantly during different time of a day due to the dynamic traffic on road segments [5].

(2) Time-dependent road networks. The shortest path query processing algorithms designed for this model have the ability to support the dynamic weight of a road segment and topology of a road network, which can change with time. This model is more realistic but more challenging. George et al. [7] proposed a time-aggregated graph, which uses time series to represent time-varying attributes. The time-aggregated graph can be used to compute the shortest path at a given start time or to find the best start time for a path that leads to the shortest travel time. In time-dependent road networks where the weight of each road segment is a function of time, problems of the time-dependent shortest-path $[5,6]$ also 
have been extensively studied. The solutions for those problems may capture the effects of periodic events (e.g., rush hours, and weekdays). However, they still cannot reflect live traffic information, which can be affected by sudden events, e.g., congestions, accidents, and road maintenance. Hence, another interesting approach for shortest path query processing is to let the server (e.g., NAVTEQ [24], and INRIX [12]) collect live traffic information and then broadcast them over radio or wireless network to clients, who listen to the live traffic index and read the relevant portion of the index for deriving the shortest path by themselves $[31,40]$.

This paper focuses on the shortest travel-time route (or path) queries in time-dependent road networks. Our work distinguishes itself from previous work in that it does not model the underlying road network based on different criteria [5-7], or broadcast live traffic information to clients $[31,40]$. Instead, it employs external Web mapping services, e.g., Google Maps, to provide the real-time route information in a road network though their user-friendly APIs.

There are some work about query processing with expensive attributes that are accessed from external Web services (e.g., [2, 3, 14, 16, 35-38]). To minimize the number of external requests, $[2,3,14]$ mainly focus on using either some cheap attributes that can be retrieved from local data sources [2,14] or sampling methods [3] to prune a whole set of objects into a smaller set of candidate objects; then, they only issue necessary external requests for retrieving candidate objects.

In [36-38], the authors proposed $k$-NN query processing algorithms that utilize grouping, direction sharing, shared query execution, pruning techniques, and parallel requesting to reduce the number of external requests to Web mapping services and provide highly accurate query answers. In [16], route logs are employed to derive tight lower/upper bounding travel times to reduce the number of external Web mapping requests for answering range and $k$-NN queries. However, none of these existing techniques focuses on how to utilize waypoints in an external route request to reduce the number of such expensive requests.

The closest work to our paper is [35], where the authors assume that the route with a longer network distance has a higher possibility of sharing its route information with others (i.e., sharing ability), and then they select waypoints in a route request only based on the network distance. In fact, this assumption is not always correct as user queries are not completely uniformly distributed in the underlying road network. Therefore, we select waypoints by sharing ability in this paper, which is computed based on the historical route information and the road network model; besides, the queries that their querying routes are independent with each other are found out for parallel processing, to reduce the query response time to users without bringing in additional external route requests.

\section{System model}

In this section, we describe our system architecture, road network model, problem definition, and the objectives of the paper. Table 1 summarizes the notation used in the paper.

\subsection{System architecture}

Figure 2 depicts the system architecture that consists of three entities: users, an LBS provider, and a Web mapping service provider (e.g., Google Maps). Users send shortest travel-time route (or path) queries with query origins and destinations to the LBS provider, 
Table 1 Summary of notation

\begin{tabular}{ll}
\hline Notation & Meaning \\
\hline$G=(V, E)$ & a road graph $G$ with node (intersection) set $V$ and edge (segment) set $E$ \\
$q_{i}=\left(o_{i}, d_{i}\right)$ & a user's shortest travel-time path query $q_{i}$ with origin $o_{i}$ and destination $d_{i}$ \\
$\mathcal{Q}$ & a set of user queries \\
$R\left(o_{i} \rightarrow d_{i}\right)$ & the shortest travel route from origin $o_{i}$ to destination $d_{i}$ \\
$l(a \rightarrow b)$ & the travel distance from location $a$ to location $b$ \\
$t(a \rightarrow b)$ & the travel time from location $a$ to location $b$ \\
$S A\left(R\left(o_{i} \rightarrow d_{i}\right), \mathcal{Q}\right)$ & the sharing ability of $R\left(o_{i} \rightarrow d_{i}\right)$ with respect to $\mathcal{Q}$ \\
$R\left(o_{i} \rightarrow d_{i}\right) \subset R\left(o_{j} \rightarrow d_{j}\right)$ & $R\left(o_{i} \rightarrow d_{i}\right)$ is the sub-route of $R\left(o_{j} \rightarrow d_{j}\right)$ \\
$S A\left(R\left(o_{i} \rightarrow d_{i}\right), \mathcal{Q}\right)=-1$ & $R\left(o_{i} \rightarrow d_{i}\right)$ is a sub-route of others \\
$w_{i}$ & a waypoint \\
$\mathcal{W}$ & an ordered waypoint set \\
$m$ & the maximum number of waypoints in a route request \\
$G_{w}=\left(V_{w}, E_{w}\right)$ & a weighted complete digraph $G_{w}$ with node (waypoint) set $V_{w}$ and edge \\
& (route) set $E_{w}$ \\
$O_{w}$ & a set of query origins \\
$D_{w}$ & a set of query destinations \\
$N_{p}$ & the number of parallel route requests submitted to the Web mapping service \\
$N D i s t(a \rightarrow b)$ & the shortest network distance from location $a$ to location $b$ \\
\hline
\end{tabular}

where the origin could be a user's current location or any other location and the destination could be any POI or location that the user wants to go or is recommended by the LBS provider. Besides turning in its own business information to a querying user, the LBS provider also returns the detailed direction information of the shortest route based on the user selected travel model (e.g., driving, walking, or cycling), which is accessed from the Web mapping service provider through external route requests.

\subsection{Road network model}

In this paper, a road map is modeled as a graph $G=(V, E)$ comprising a set $V$ of vertices with a set $E$ of edges, where each road segment is an edge and each intersection of road segments is a vertex. For instance, Fig. 3a depicts a real road map that is modeled as an undirected graph (Fig. 3b), where an edge represents a road segment (e.g., $v_{1} v_{2}$ and $v_{1} v_{5}$ ) and a square represents an intersection of road segments (e.g., $v_{1}$ and $v_{2}$ ). As the weight of

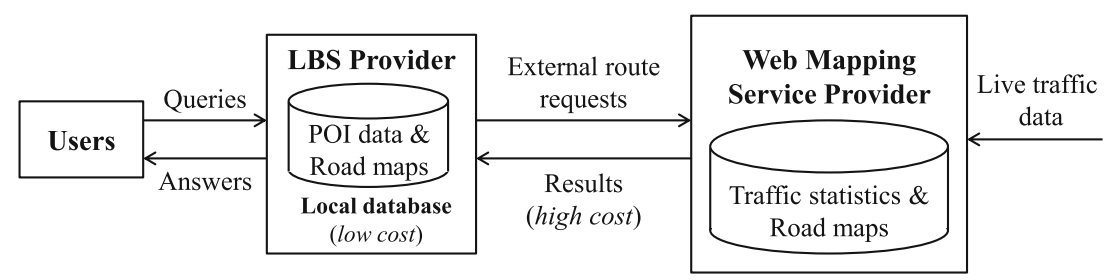

Fig. 2 System architecture 


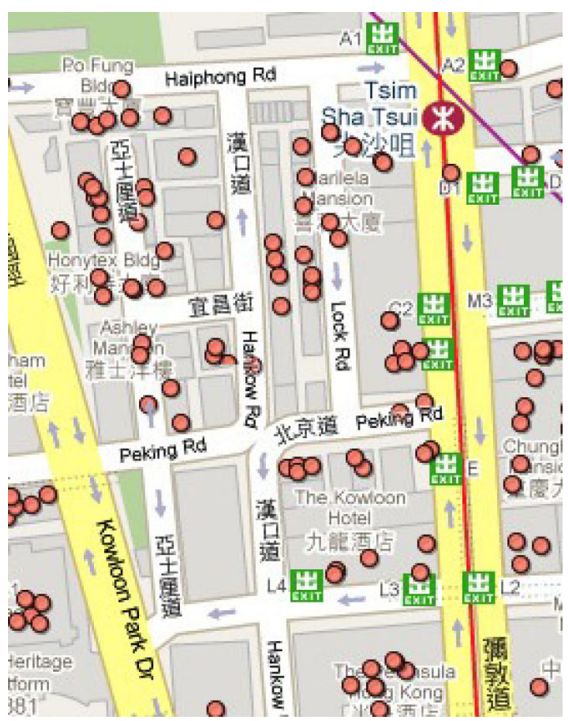

(a) A road map

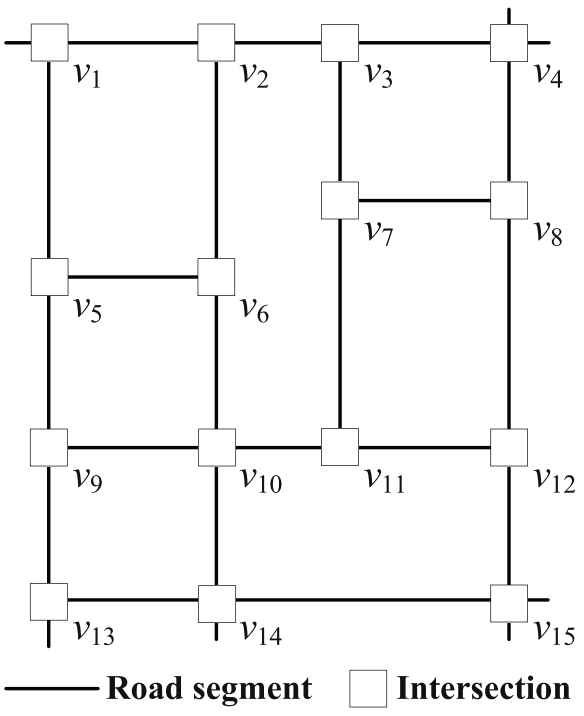

(b) A graph model

Fig. 3 Road network model

each edge is static (i.e., the network distance), $G$ cannot provide real-time travel-time-based route information.

\subsection{Our problem and objectives}

Given a set of user queries $\mathcal{Q}=\left\{q_{1}, q_{2}, \ldots, q_{n}\right\}$ arrived concurrently or within a short time period at the LBS provider, and each user query $q_{i}$ is in a form of $\left(o_{i}, d_{i}\right)$, where $o_{i}$ and $d_{i}$ are query origin and destination, respectively, the LBS provider returns the shortest travel-time route with the detailed direction information from $o_{i}$ to $d_{i}$ for each user query $q_{i}$ based on live traffic conditions, which are retrieved from the Web mapping service provider through external route requests.

Since there are two critical limitations to access the travel route information from a Web mapping service as stated above, i.e., high cost and usage limits, our objectives are to reduce the number of external route requests issued by the LBS provider and the query response time to its users.

\section{Direction sharing optimization}

\subsection{Optimal sub-route property}

For an external route request with an origin and a destination, the Web mapping service will return the shortest travel-time route from the origin to the destination with detailed turn-byturn direction information. For example, if the LBS provider sends an external route request to the Web mapping service to retrieve the travel route and time information from origin $o_{3}$ 


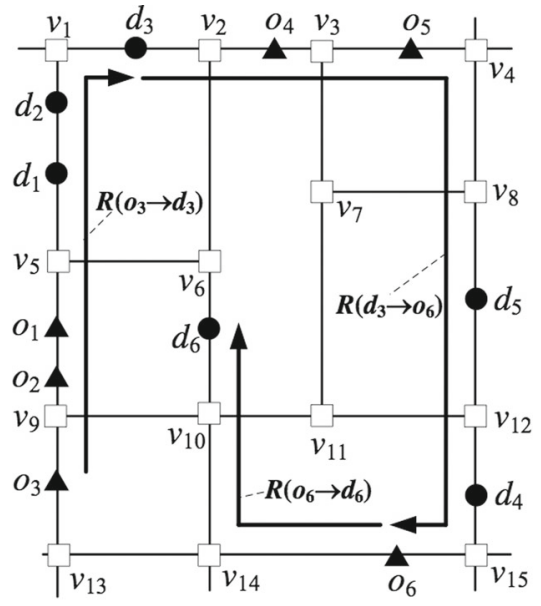

$\Delta$ Origin ODestination $\square$ Intersection

(a) Route information

\begin{tabular}{|c|c|c|}
\hline Query & $\begin{array}{c}\text { Sharing } \\
\text { ability }\end{array}$ & $\begin{array}{c}\text { Shared } \\
\text { queries }\end{array}$ \\
\hline$q_{1}=\left(o_{1}, d_{1}\right)$ & $-1^{*}$ & - \\
\hline$q_{2}=\left(o_{2}, d_{2}\right)$ & $-1^{*}$ & - \\
\hline $\boldsymbol{q}_{3}=\left(\boldsymbol{o}_{\mathbf{3}}, d_{3}\right)$ & $\mathbf{3}$ & $\boldsymbol{q}_{\mathbf{1}}, \boldsymbol{q}_{\mathbf{2}}, \boldsymbol{q}_{\mathbf{3}}$ \\
\hline $\boldsymbol{q}_{\mathbf{4}}=\left(\boldsymbol{o}_{\mathbf{4}}, \boldsymbol{d}_{\mathbf{4}}\right)$ & $\mathbf{2}$ & $\boldsymbol{q}_{\mathbf{4}}, \boldsymbol{q}_{\mathbf{5}}$ \\
\hline$q_{5}=\left(o_{5}, d_{5}\right)$ & $-1^{*}$ & - \\
\hline $\boldsymbol{q}_{\mathbf{6}}=\left(\boldsymbol{o}_{\mathbf{6}}, \boldsymbol{d}_{\mathbf{6}}\right)$ & $\mathbf{1}$ & $\boldsymbol{q}_{\mathbf{6}}$ \\
\hline
\end{tabular}

* -1 means the query route can share the direction information with others, so there is no need to compute its sharing ability any more.

(b) Sharing ability

Fig. 4 Route information and sharing ability for $\mathcal{Q}=\left\{q_{1}, q_{2}, q_{3}, q_{4}, q_{5}, q_{6}\right\}$

(represented by a triangle) to destination $d_{3}$ (represented by a circle) as shown in Fig. 4a, the service returns $R\left(o_{3} \rightarrow d_{3}\right)=\left\{\left\langle l\left(o_{3} \rightarrow v_{9}\right), t\left(o_{3} \rightarrow v_{9}\right)\right\rangle,\left\langle l\left(v_{9} \rightarrow v_{5}\right), t\left(v_{9} \rightarrow\right.\right.\right.$ $\left.\left.\left.v_{5}\right)\right\rangle,\left\langle l\left(v_{5} \rightarrow v_{1}\right), t\left(v_{5} \rightarrow v_{1}\right)\right\rangle,\left\langle l\left(v_{1} \rightarrow d_{3}\right), t\left(v_{1} \rightarrow d_{3}\right)\right\rangle\right\}$, where $l(a \rightarrow b)$ and $t(a \rightarrow$ $b)$ are the travel distance and time from location $a$ to location $b$, respectively. For simplicity, we denote $R\left(o_{3} \rightarrow d_{3}\right)=\left\{o_{3} \rightarrow v_{9} \rightarrow v_{5} \rightarrow v_{1} \rightarrow d_{3}\right\}$.

Shortest routes exhibit the optimal sub-route property [4], i.e., every sub-route of the shortest route is also the shortest sub-route (see Lemma 1). In other words, the shortest route $R\left(o_{i} \rightarrow d_{i}\right)$ returned from the Web mapping service for a query $q_{i}=\left(o_{i}, d_{i}\right)$ can be shared with another query $q_{j}=\left(o_{j}, d_{j}\right)$, if both $o_{j}$ and $d_{j}$ are located in the route, ${ }^{1}$ i.e., $o_{j} \in R\left(o_{i} \rightarrow d_{i}\right)$ and $d_{j} \in R\left(o_{i} \rightarrow d_{i}\right)$; thus, there is no need for the LBS provider to issue an external route request for $q_{j}=\left(o_{j}, d_{j}\right)$ any more. As a result, the number of external route requests can be reduced.

Lemma 1 (Optimal sub-route property) Let $R\left(o_{i} \rightarrow d_{i}\right)=\left\{o_{i} \rightarrow \cdots \rightarrow o_{j} \rightarrow \cdots \rightarrow\right.$ $\left.d_{j} \rightarrow \cdots \rightarrow d_{i}\right\}$ be the shortest travel-time route for a query with origin location $o_{i}$ to destination location $d_{i} . R\left(o_{j} \rightarrow d_{j}\right)=\left\{o_{j} \rightarrow \cdots \rightarrow d_{j}\right\}$ is also the shortest travel-time route from $o_{j}$ to $d_{j}$.

Proof See the proof of Lemma 24.1 in [4].

For example, only one external route request is needed instead of three to access the travel route information for $q_{1}=\left(o_{1}, d_{1}\right), q_{2}=\left(o_{2}, d_{2}\right)$, and $q_{3}=\left(o_{3}, d_{3}\right)$, as the travel

\footnotetext{
${ }^{1}$ If $R\left(o_{i} \rightarrow d_{i}\right)$ is directional, $o_{j}$ and $d_{j}$ should locate in the route in order.
} 
route information of $q_{1}$ (i.e., $R\left(o_{1} \rightarrow d_{1}\right)$ ) and $q_{2}$ (i.e., $R\left(o_{2} \rightarrow d_{2}\right)$ ) can be shared with $q_{3}$ (i.e., $\left.R\left(o_{3} \rightarrow d_{3}\right)\right)$ to find their travel route information, as shown in Fig. $4 \mathrm{a}$.

\subsection{Sharing ability}

\subsubsection{Definition}

To reflect the possibility of sharing the direction information of a route with others, we formally define its sharing ability as follows:

Definition 1 (Sharing ability) Let $\mathcal{Q}$ be a set of user queries, and $R\left(o_{i} \rightarrow d_{i}\right)$ be the shortest travel-time route from origin location $o_{i}$ to destination location $d_{i}$. The sharing ability of $R\left(o_{i} \rightarrow d_{i}\right)$ with respect to $\mathcal{Q}$ (denoted as $S A\left(R\left(o_{i} \rightarrow d_{i}\right), \mathcal{Q}\right)$ ) is the number of queries in $\mathcal{Q}$, where the direction information of $R\left(o_{i} \rightarrow d_{i}\right)$ can be shared with these queries, i.e.,

$$
S A\left(R\left(o_{i} \rightarrow d_{i}\right), \mathcal{Q}\right)=\left|\left\{q_{j}=\left(o_{j}, d_{j}\right) \in \mathcal{Q} \mid R\left(o_{j} \rightarrow d_{j}\right) \subset R\left(o_{i} \rightarrow d_{i}\right)\right\}\right| .
$$

As an example depicted in Fig. 4a, where $\mathcal{Q}=\left\{q_{1}, q_{2}, q_{3}, q_{4}, q_{5}, q_{6}\right\}$ and $R\left(o_{1} \rightarrow\right.$ $\left.d_{1}\right) \subset R\left(o_{2} \rightarrow d_{2}\right) \subset R\left(o_{3} \rightarrow d_{3}\right)$, i.e., three queries $\left(q_{1}, q_{2}, q_{3}\right)$ in $\mathcal{Q}$ can share the direction information of $R\left(o_{3} \rightarrow d_{3}\right)$, the sharing ability of $R\left(o_{3} \rightarrow d_{3}\right)$ with respect to $\mathcal{Q}$ is 3, i.e., $S A\left(R\left(o_{3} \rightarrow d_{3}\right), \mathcal{Q}\right)=3$. Since $q_{1}$ and $q_{2}$ can share the direction information of $R\left(o_{3} \rightarrow d_{3}\right)$, we set the value of $S A\left(R\left(o_{1} \rightarrow d_{1}\right), \mathcal{Q}\right)$ and $S A\left(R\left(o_{2} \rightarrow d_{2}\right), \mathcal{Q}\right)$ to -1 as a flag to show that there is no need to compute them any more. Similarly, we can compute the sharing ability of $q_{4}, q_{5}$ and $q_{6}$, which are shown in Fig. 4b. Therefore, three external route requests are needed to access the direction information for all the queries in $\mathcal{Q}$ instead of six.

Since the sharing ability can reflect the possibility of sharing the direction information of a route with others, to minimize the query response time to users and the number of external route requests, the LBS provider should process queries in $\mathcal{Q}$ in a non-increasing order based on their sharing abilities to fully utilize the direction sharing optimization. In the running example, the query answer of three queries (i.e., $q_{1}, q_{2}, q_{3}$ ) can be retrieved at the same time if $q_{3}$ is processed first, while only one query answer can be retrieved if $q_{6}$ is the first to be processed.

\subsubsection{Calculation}

However, the current direction information between two locations is unknown until the LBS provider issues an external route request to the Web mapping service and gets the result. Only knowing the road network model $G$ and the origins and destinations of queries in $\mathcal{Q}$, it would be impossible to calculate the exact sharing ability of a route.

To this end, we plan to utilize historical route information (as [16, 29]), which are stored in the local database at the LBS provider when retrieved from the Web mapping service, to approximately reflect current route information and then compute route sharing abilities. Only when there is no historical direction information of a route in the local database, we calculate it based on the road network model $G$ by employing Dijkstra algorithm (or $A^{*}$ algorithm). For example, $R\left(o_{3} \rightarrow d_{3}\right)$ can be accessed from the local database if there is historical direction information from $v_{13}$ to $v_{2}$, i.e., $v_{13} \rightarrow v_{9} \rightarrow v_{5} \rightarrow v_{1} \rightarrow v_{2}$; otherwise, we compute the shortest network distance path from $o_{3}$ to $d_{3}$ based on $G$, and take the path as $R\left(o_{3} \rightarrow d_{3}\right)$. 


\subsubsection{Algorithm}

Algorithm 1 depicts the pseudo code of computing the sharing ability of the route from origin $o_{i}$ to destination $d_{i} . R\left(o_{i} \rightarrow d_{i}\right)$ is retrieved from the historical route information in local database or computed based on $G$ first. Then, for each query $q_{j}=\left(o_{j}, d_{j}\right)$ in $\mathcal{Q}$, if both $o_{j}$ and $d_{j}$ locate in $R\left(o_{i} \rightarrow d_{i}\right)$, the sharing ability of $R\left(o_{i} \rightarrow d_{i}\right)$ with respect to $\mathcal{Q}$ is increased by one based on Definition 1, and the sharing ability of $R\left(o_{j} \rightarrow d_{j}\right)$ with respect to $\mathcal{Q}$ is set to -1 as a flag to show that there is no need to compute it any more.

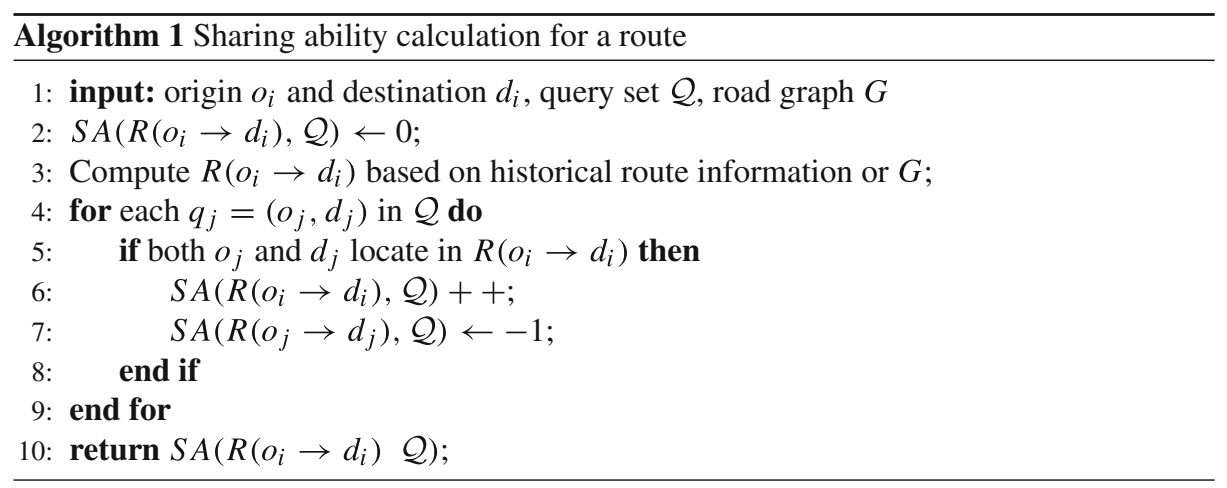

\subsection{Parallel processing for independent routes}

Web mapping services can handle a large number of route requests at the same time; hence, another good way to further reduce the user query response time is the LBS provider issues external route requests to the Web mapping service in parallel. In order not to bring in additional requests to Web mapping services, route requests issued in parallel should be independent with each other (see Definition 2).

In the running example, the routes of $q_{3}$ and $q_{4}$ are independent. However, the routes of $q_{2}$ and $q_{3}$ are not, and an extra route request is needed if the routes of $q_{2}$ and $q_{3}$ are retrieved at the same time.

Definition 2 (Independent routes) Two routes $R\left(o_{i} \rightarrow d_{i}\right)$ and $R\left(o_{j} \rightarrow d_{j}\right)$ are independent if they are not the sub-route of each other, i.e., $R\left(o_{i} \rightarrow d_{i}\right) \not \subset R\left(o_{j} \rightarrow d_{j}\right)$ and $R\left(o_{j} \rightarrow d_{j}\right) \not \subset R\left(o_{i} \rightarrow d_{i}\right)$.

Definition 3 (Independent queries) Queries are independent if their querying routes are independent with each other.

To this end, independent queries (see Definition 3) in $\mathcal{Q}$ should be found for parallel processing. In fact, those independent queries have been detected out after the calculation of sharing ability, i.e., queries are independent if their sharing abilities are not equal to -1 (see Lemma 2). 
Lemma 2 Given a user query set $\mathcal{Q}, \forall q_{i}, q_{j} \in \mathcal{Q}$, if both $S A\left(R\left(o_{i} \rightarrow d_{i}\right), \mathcal{Q}\right) \neq-1$ and $S A\left(R\left(o_{j} \rightarrow d_{j}\right), \mathcal{Q}\right) \neq-1$, then $R\left(o_{i} \rightarrow d_{i}\right)$ and $R\left(o_{j} \rightarrow d_{j}\right)$ are independent, where $R\left(o_{i} \rightarrow d_{i}\right)$ and $R\left(o_{j} \rightarrow d_{j}\right)$ are the querying route of $q_{i}$ and $q_{j}$, respectively.

Proof As presented in Section 4.2, $S A\left(R\left(o_{i} \rightarrow d_{i}\right), \mathcal{Q}\right) \neq-1$ means $R\left(o_{i} \rightarrow d_{i}\right)$ is not the sub-route of any other querying routes, i.e., $R\left(o_{i} \rightarrow d_{i}\right) \not \subset R\left(o_{j} \rightarrow d_{j}\right)$. Similarly, $R\left(o_{j} \rightarrow d_{j}\right) \not \subset R\left(o_{i} \rightarrow d_{i}\right)$. Based on Definition 2, $R\left(o_{i} \rightarrow d_{i}\right)$ and $R\left(o_{j} \rightarrow d_{j}\right)$ are independent.

Take Fig. 4 for example, the routes of $q_{3}, q_{4}$ and $q_{6}$ are not the sub-route of any other querying routes, so they are independent with each other and can be retrieved at the same time by the LBS provider with three external route requests.

\section{Route waypoint optimization}

\subsection{Definition}

Most of existing Web mapping services support adding multiple waypoints in a route request. Waypoints alter a route by routing it through the specified locations (see Definition 4). However, most of queries issued by users only seek for the shortest travel-time route information from their own origins to corresponding destinations, i.e., only two waypoints, which is less than the maximum allowed number of waypoints provided by Web mapping service providers (see Table 2). For example, Google Maps allows up to 8 intermediate waypoints in a route request for evaluation users and 23 intermediate waypoints for premier users [9]. On the other hand, there is usually a limit or charge on the number of route requests to a Web mapping service.

Definition 4 (Waypoint) A waypoint is a specified location that is used for navigation purposes. Given a route request with a set of ordered waypoints $\mathcal{W}=\left\{w_{1}, w_{2}, \cdots, w_{m}\right\}$, where $w_{1}$ and $w_{m}$ are the origin and destination locations, respectively, and the others are intermediate locations, the Web mapping service will return the detailed direction information from $w_{1}$ to $w_{2}$ to $\cdots$ to $w_{m}$ as a result for the route request, i.e., $R\left(w_{1} \rightarrow w_{2} \rightarrow\right.$ $\left.\cdots \rightarrow w_{m}\right)$, and the sub-route $R\left(w_{i} \rightarrow w_{i+1}\right)(1 \leq i \leq m-1)$ between any two successive waypoints in the result.

Table 2 The maximum number of waypoints in a route request supported by three popular Web mapping services (Accessed on August 7, 2016)

\begin{tabular}{ll}
\hline Web mapping services & No. of waypoints \\
\hline Google Maps for evaluation users & 8 \\
Google Maps for premier users & 23 \\
Bing Maps & 25 \\
MapQuest Maps & 50
\end{tabular}


It is unwise for the LBS provider to simply issue external route requests to the Web mapping service provider for each query in $\mathcal{Q}$ separately without utilizing waypoints. Thus, a more efficient way is composing multiple independent user queries in $\mathcal{Q}$ as one external route request by taking the origins and destinations of those queries as waypoints.

For the example depicted in Fig. 4, processing each query in $\mathcal{Q}$ separately by using the direction sharing optimization requires three external route requests, as discussed in Section 4.3. Only one request with ordered waypoints $\mathcal{W}=\left\{o_{3}, d_{3}, o_{6}, d_{6}\right\}$ is needed to access the travel route information for all the queries in $\mathcal{Q}$. This is because the returned route $R\left(o_{3} \rightarrow d_{3} \rightarrow o_{6} \rightarrow d_{6}\right)$ consists of $R\left(o_{3} \rightarrow d_{3}\right), R\left(d_{3} \rightarrow o_{6}\right)$ and $R\left(o_{6} \rightarrow d_{6}\right)$, which can be used for the query answers of $q_{1} q_{2} q_{3}, q_{4} q_{5}$, and $q_{6}$, respectively.

\subsection{Problem modeling}

To minimize the number of external route requests, the LBS provider should make full utilization of the direction sharing and waypoints provided by Web mapping services. The optimal ordered waypoint set $\mathcal{W}=\left\{w_{1}, \cdots, w_{m}\right\}$ should be determined for an external route request to maximize the possibility of sharing its direction information with other queries in $\mathcal{Q}$, where $m$ is the maximum number of waypoints in a route request supported by Web mapping services. In other words, we should maximize the sharing ability of $R\left(w_{1} \rightarrow\right.$ $\cdots \rightarrow w_{m}$ ) with respect to $\mathcal{Q}$ by maximizing the sharing ability of the sub-routes in $\mathcal{W}$ with respect to $\mathcal{Q}$; hence,

$$
S A\left(R\left(w_{1} \rightarrow \cdots \rightarrow w_{m}\right), \mathcal{Q}\right)=\sum_{i=1}^{m-1} S A\left(R\left(w_{i} \rightarrow w_{i+1}\right), \mathcal{Q}\right) .
$$

To find the optimal $R\left(w_{1} \rightarrow \cdots \rightarrow w_{m}\right)$, we first model the origins and destinations of the independent queries in $\mathcal{Q}$ and all routes between them as a weighted complete digraph $G_{w}=\left(V_{w}, E_{w}\right)$ comprising a set $V_{w}$ of vertices, and a set $E_{w}$ of weighted directed edges. The set $V_{w}$ is composed by origins and destinations of all independent queries in $\mathcal{Q}$. The set $E_{w}$ consists of directed routes between every pair of distinct vertices in $V_{w}$. The weight of each edge in $E_{w}$ is represented by the sharing ability of its corresponding route, i.e., weight $\left(v_{i} v_{j}\right)=S A\left(R\left(v_{i} \rightarrow v_{j}\right), \mathcal{Q}\right)\left(v_{i}, v_{j} \in V_{w}\right)$.

After modeling the independent queries in $\mathcal{Q}$ as a weighted complete digraph $G_{w}=$ $\left(V_{w}, E_{w}\right)$, we should find a waypoint set $\mathcal{W}=\left\{w_{1}, \cdots, w_{m}\right\}$ for $\mathcal{Q}$ such that the route $R\left(w_{1} \rightarrow \cdots \rightarrow w_{m}\right)$ will result in the best sharing ability. We can transform this problem into how to find a simple path in $G_{w}$ with the largest weight via at most $m$ vertices, which is a MAX SNP-hard problem (see Lemma 3), i.e., there is no polynomialtime algorithm that can even find a constant factor approximation for the problem unless $\mathrm{P}=\mathrm{NP}[13]$.

Lemma 3 Finding a simple path in a weighted complete digraph $G_{w}=\left(V_{w}, E_{w}\right)$ with the largest weight via at most $m$ vertices is a MAX SNP-hard problem.

Proof Consider a special case of the problem: set the value of $m$ to the number of vertices in $G_{w}$, i.e., $m=\left|V_{w}\right|$. Under this condition, the problem is reduced to the maximum- 
weight traveling-salesman (Max TSP) problem, also known informally as the taxicab rip-off problem, which is a MAX SNP-hard problem [10]. Thus, the lemma is proved.

\subsection{Waypoint selection}

In this work, we aim to find the best set of waypoints in an external route request for all the queries in $\mathcal{Q}$ by designing a greedy algorithm with performance guarantee. The algorithm guarantees that at least $\left\lfloor\frac{m}{2}\right\rfloor$ queries in $\mathcal{Q}$ can share the direction information of the returned route from Web mapping services, which is specified by the selected waypoints, and tries to maximize the direction sharing power of the route at the same time. The details of the greedy algorithm are as follows.

\subsubsection{The greedy algorithm}

Given a query set $\mathcal{Q}$ and the maximum number of waypoints $m$, Algorithm 2 depicts the pseudo code of a greedy algorithm for finding the best waypoint set $\mathcal{W}$ for an external route request. It consists of three main steps:

Step 1: Preparation Initially, the waypoint set $\mathcal{W}$, the origin set $O_{w}$ (for storing query origin location $o_{i}$ ), and the destination set $D_{w}$ (for storing query destination location $d_{i}$ ) are set to empty. For each query $q_{i}=\left(o_{i}, d_{i}\right)$ in $\mathcal{Q}$, the sharing ability of its querying route with respect to $\mathcal{Q}$ (i.e., $S A\left(R\left(o_{i} \rightarrow d_{i}\right), \mathcal{Q}\right)$ is calculated by Algorithm 1 . Then, the query origins and destinations of independent routes (i.e., $S A\left(R\left(o_{i} \rightarrow d_{i}\right), \mathcal{Q}\right)>0$, based on Lemma 2) are inserted into $O_{w}$ and $D_{w}$, respectively. (Lines 3 to 13 in Algorithm 2)

Step 2: Initial waypoint selection In this step, the origin $o_{l}$ and destination $d_{l}$ of the query $q_{l}$ with the largest sharing ability are selected. $o_{l}$ is inserted into $\mathcal{W}$ as the first waypoint, and it is removed from $O_{w}$. If the number of waypoint in $\mathcal{W}$ (i.e., $|\mathcal{W}|$ ) is less than $m, d_{l}$ is also inserted into $\mathcal{W}$ as the second waypoint and removed from $D_{w}$. The algorithm proceeds to the next step if $|\mathcal{W}|<m$ and $O_{w}$ is not empty. (Lines 15 to 19 in Algorithm 2)

Step 3: Greedy waypoint selection In this step, $d_{l}$ is the last waypoint inserted into $\mathcal{W}$. The next waypoint $o_{t}$ is selected from all the origins in $O_{w}$ such that $R\left(d_{l} \rightarrow o_{t} \rightarrow d_{t}\right)$ has the highest sharing ability. If the locations of $d_{l}$ and $o_{t}$ are the same, there is no need to insert $o_{t}$ into $\mathcal{W}$ anymore, as $d_{l}$ not only is the query destination of $q_{l}$, but also can be taken the query origin of $q_{t}$. For each independent query $q_{i}=\left(o_{i}, d_{i}\right)$ in $\mathcal{Q}$, if both $o_{i}$ and $d_{i}$ locate in $R\left(d_{l} \rightarrow o_{t}\right)$, i.e., $R\left(o_{i} \rightarrow d_{i}\right) \subset R\left(d_{l} \rightarrow o_{t}\right), o_{i}$ and $d_{i}$ are removed from $O_{w}$ and $D_{w}$, respectively, as the retrieved direction information of $R\left(d_{l} \rightarrow o_{t}\right)$ may be utilized as the answer of $q_{i}$. If $|\mathcal{W}|<m$, the corresponding destination $d_{t}$ of $o_{t}$ is also added to $\mathcal{W}$ and removed from $D_{w}$. This step is repeated until $|\mathcal{W}|=m$ or both the origin and destination sets (i.e., $O_{w}$ and $D_{w}$ ) become empty. (Lines 21 to 41 in Algorithm 2) 


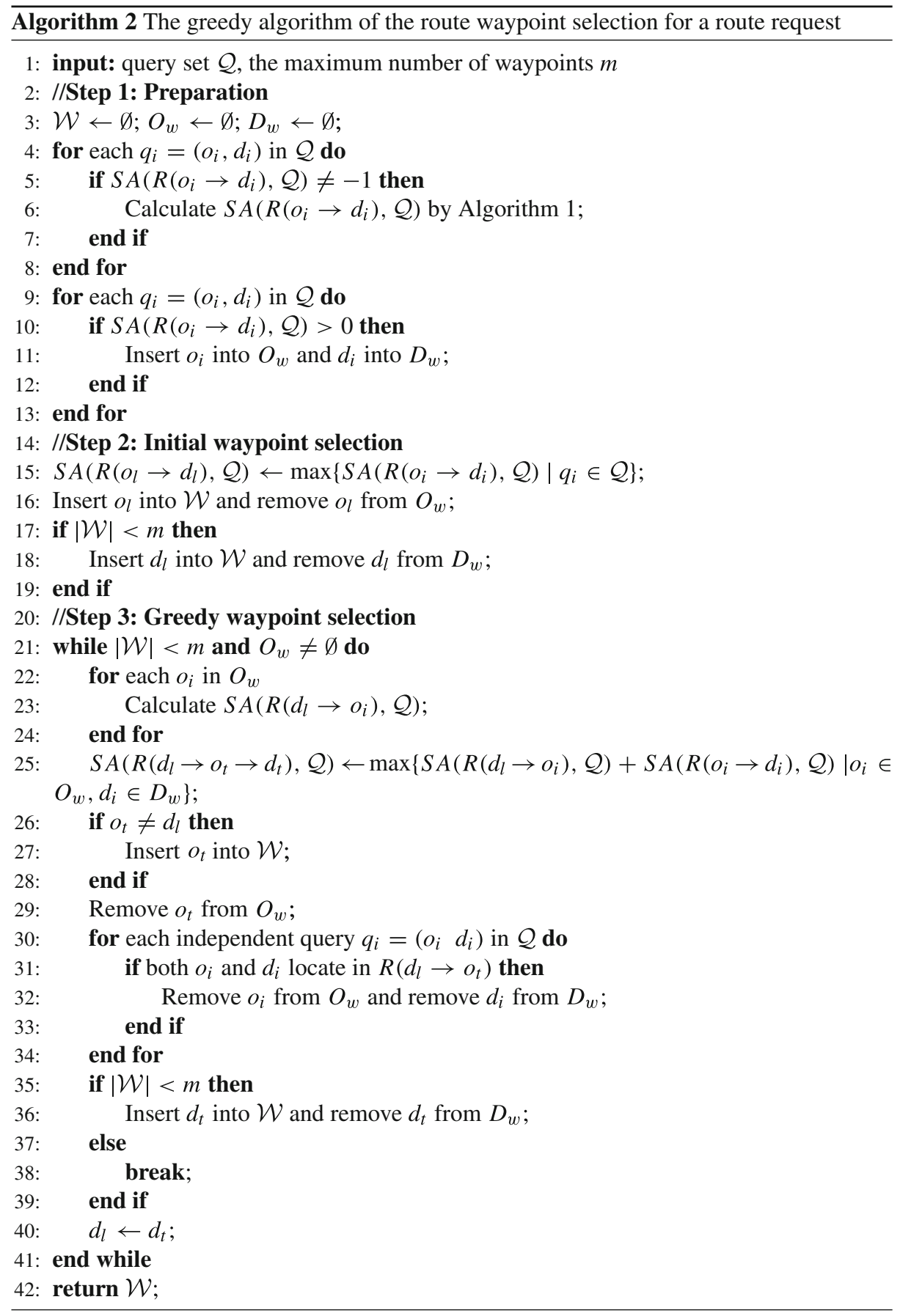

Based on Algorithm 2, we have the following lemma: 
Lemma 4 At least $\left\lfloor\frac{m}{2}\right\rfloor$ queries in $\mathcal{Q}$ can share the direction information of the returned route from Web mapping services specified by waypoints in $\mathcal{W}$ determined by Algorithm 2, where $m$ is the maximum number of waypoints in a route request.

Proof As shown in Lines 17 to 19 and Lines 35 to 39 of Algorithm 2, when a query origin $o_{i}$ is selected into the waypoint set $\mathcal{W}$, its corresponding query destination $d_{i}$ is also selected if $o_{i}$ is not the last waypoint; therefore, at least $\left\lfloor\frac{m}{2}\right\rfloor$ pairs of query origins and destinations are in the returned route from Web mapping services specified by waypoints in $\mathcal{W}$. Thus, the lemma is proved.

In summary, even though Algorithm 2 cannot select optimal waypoints in a route request as the target problem is MAX SNP-hard based on Lemma 3, it still can guarantee that at least $\left\lfloor\frac{m}{2}\right\rfloor$ pairs of query origins and destinations are selected, i.e., at least $\left\lfloor\frac{m}{2}\right\rfloor$ queries in $\mathcal{Q}$ can be processed in one route request (see Lemma 4), and it tries to maximize the direction sharing optimization of the route at the same time.

\subsubsection{Example}

Table 3 gives an example of Algorithm 2 in detail, where $\mathcal{Q}=\left\{q_{1}, q_{2}, q_{3}, q_{4}, q_{5}, q_{6}\right\}$ (Fig. 4), and the maximum number of waypoints in an external route request is four, i.e., $m=4$. When $|\mathcal{W}|=m$ or $O_{w}$ and $D_{w}$ becomes empty, the algorithm returns $\mathcal{W}=\left\{o_{3}, d_{3}\right.$, $\left.o_{6}, d_{6}\right\}$ and terminates here.

Table 3 Example of Algorithm 2 (input: $\mathcal{Q}=\left\{q_{1}, q_{2}, q_{3}, q_{4}, q_{5}, q_{6}\right\}, m=4$ )

\begin{tabular}{|c|c|c|c|c|c|c|}
\hline Step & Lines & $\mathcal{W}$ & $O_{w}$ & $D_{w}$ & $d_{l}$ & Checking/Actions \\
\hline $\begin{array}{l}\text { Step 1: } \\
\text { Preparation }\end{array}$ & $\begin{array}{l}\text { Lines } 3 \\
\text { to } 13\end{array}$ & & $\left\{o_{3}, o_{4}, o_{6}\right\}$ & $\left\{d_{3}, d_{4}, d_{6}\right\}$ & - & $\begin{array}{l}\text { Calculate the sharing ability of each } \\
\text { query in } \mathcal{Q} \text { (Fig. } 4 \text { ) } \Rightarrow q_{3}, q_{4}, q_{6} \text { are } \\
\text { independent } \Rightarrow \text { their origins and desti- } \\
\text { nations are inserted into } O_{w} \text { and } D_{w} \text {, } \\
\text { respectively }\end{array}$ \\
\hline $\begin{array}{l}\text { Step 2: } \\
\text { Initial } \\
\text { waypoint } \\
\text { selection }\end{array}$ & $\begin{array}{l}\text { Lines } 15 \\
\text { to } 19\end{array}$ & $\left\{o_{3}, d_{3}\right\}$ & $\left\{o_{4}, o_{6}\right\}$ & $\left\{d_{4}, d_{6}\right\}$ & $d_{3}$ & $\begin{array}{l}q_{3} \text { owns the largest sharing ability } \Rightarrow o_{3} \\
\text { and } d_{3} \text { are selected into } \mathcal{W} \text { and removed } \\
\text { from } O_{w} \text { and } D_{w} \text {, respectively; } d_{l}=d_{3}\end{array}$ \\
\hline \multirow[t]{3}{*}{$\begin{array}{l}\text { Step 3: } \\
\text { Greedy } \\
\text { waypoint } \\
\text { selection }\end{array}$} & $\begin{array}{l}\text { Lines } 21 \\
\text { to } 29\end{array}$ & $\left\{o_{3}, d_{3}, o_{6}\right\}$ & $\left\{o_{4}\right\}$ & $\left\{d_{4}, d_{6}\right\}$ & $d_{3}$ & $\begin{array}{l}\text { Calculate that } S A\left(R\left(d_{3} \rightarrow o_{4}\right), \mathcal{Q}\right)= \\
0 \text { and } S A\left(R\left(d_{3} \rightarrow o_{6}\right), \mathcal{Q}\right)=2 \Rightarrow \\
S A\left(R\left(d_{3} \rightarrow o_{4} \rightarrow d_{4}\right), \mathcal{Q}\right)=2< \\
S A\left(R\left(d_{3} \rightarrow o_{6} \rightarrow d_{6}\right), \mathcal{Q}\right)=3, \text { and } \\
o_{6} \neq d_{3} \Rightarrow o_{6} \text { is inserted into } \mathcal{W} \text { and } \\
\text { removed from } O_{w}\end{array}$ \\
\hline & $\begin{array}{l}\text { Lines } 30 \\
\text { to } 34\end{array}$ & $\left\{o_{3}, d_{3}, o_{6}\right\}$ & $\emptyset$ & $\left\{d_{6}\right\}$ & $d_{3}$ & $\begin{array}{l}\text { Both } o_{4} \text { and } d_{4} \text { locate in } R\left(d_{3} \rightarrow o_{6}\right) \\
\Rightarrow o_{4} \text { and } d_{4} \text { are removed from } O_{w} \text { and } \\
D_{w} \text {, respectively }\end{array}$ \\
\hline & $\begin{array}{l}\text { Lines } 35 \\
\text { to } 40\end{array}$ & $\left\{o_{3}, d_{3}, o_{6}, d_{6}\right\}$ & $\emptyset$ & $\emptyset$ & $d_{6}$ & $\begin{array}{l}|\mathcal{W}|<4 \Rightarrow d_{6} \text { is insert into } \mathcal{W} \text { and } \\
\text { removed from } D_{w} ; d_{l} \leftarrow d_{6}\end{array}$ \\
\hline
\end{tabular}




\section{Algorithm with the proposed optimizations}

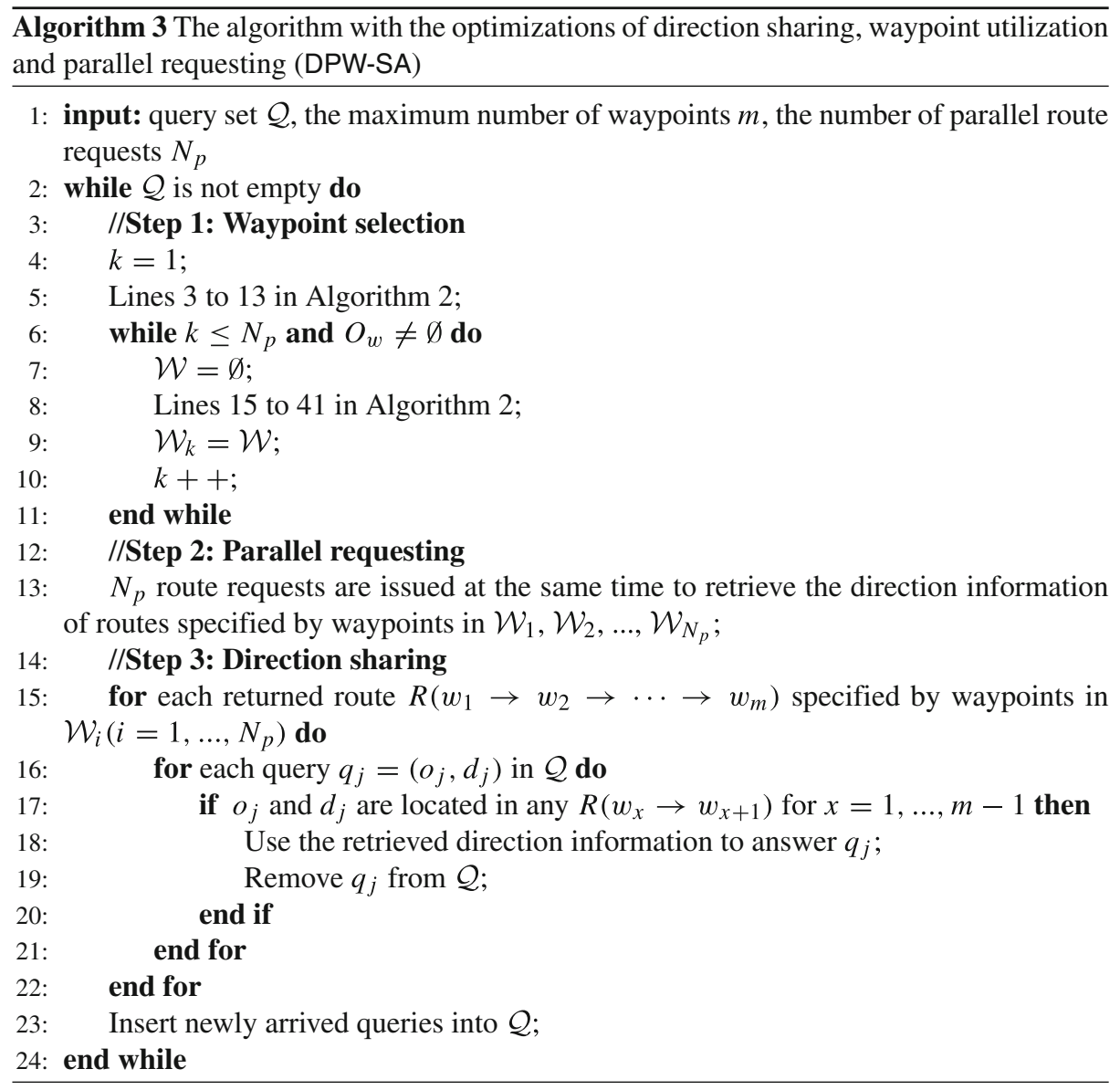

Algorithm 3 (denoted as DPW-SA) depicts the pseudo code of the algorithm with the optimizations of Direction sharing, Parallel requesting, and Waypoints selected based on the Sharing Ability, to minimize the number of route requests and the user query response time. The algorithm has the following three steps:

Step 1: Waypoint selection Algorithm 2 is only to find the best set of waypoints in one external route request. To support parallel requesting, Algorithm 3 extends Algorithm 2 by adding a while loop in it to determine a number of waypoint sets, i.e., $\mathcal{W}_{1}, \mathcal{W}_{2}, \ldots, \mathcal{W}_{N_{p}}$, where $N_{p}$ is the number of route requests submitted to Web mapping services at the same time. (Lines 4 to 11 in Algorithm 3) 
Step 2: Parallel requesting Then, the LBS provider issues $N_{p}$ route requests to Web mapping services at the same time to retrieve the direction information of those routes. Each route request is specified by each waypoint set $\mathcal{W}_{i}\left(1<i<N_{p}\right)$. (Line 13 in Algorithm 3)

Step 3: Direction sharing When the direction information of a route request is returned, i.e., $R\left(w_{1} \rightarrow w_{2} \rightarrow \cdots \rightarrow w_{m}\right)$, for each query $q_{j}=\left(o_{j}, d_{j}\right)$ in $\mathcal{Q}$, if both $o_{j}$ and $d_{j}$ are located in any $R\left(w_{x} \rightarrow w_{x+1}\right)$ for $x=1, \cdots, m-1$, the retrieved direction information can be used to answer $q_{j}$ and $q_{j}$ is removed from $\mathcal{Q}$. (Lines 15 to 22 in Algorithm 3)

The algorithm repeatedly deals with the remaining and newly arrived queries in $\mathcal{Q}$ until $\mathcal{Q}$ becomes empty.

\section{Performance evaluation}

\subsection{The baseline approach}

To the best of our knowledge, there is no other existing work about taking advantage of waypoints in route requests to reduce the number of external route requests and the query response time for shortest travel-time path query processing, except for our previous work [35].

In [35], waypoints are selected based on the network distance instead of sharing ability, e.g., the two initial waypoints $o_{l}$ and $d_{l}$ are selected based on NDist $\left(o_{l} \rightarrow d_{l}\right)=$ $\max \left\{N \operatorname{Dist}\left(o_{i} \rightarrow d_{i}\right) \mid q_{i} \in \mathcal{Q}\right\}$, and the next waypoint $o_{t}$ is selected based on $N \operatorname{Dist}\left(d_{l} \rightarrow o_{t}\right)=\max \left\{N \operatorname{Dist}\left(d_{l} \rightarrow o_{i}\right) \mid o_{i} \in O_{w}\right\}$, where $N \operatorname{Dist}(a \rightarrow b)$ stands for the shortest network distance from location $a$ to location $b$; therefore, independent queries in $\mathcal{Q}$ can not be found out only based on the network distance. Besides, parallel requesting are also not equipped in [35] for reducing the user query response time.

To this end, we extend the algorithm proposed in [35] with parallel requesting and take it as the baseline algorithm (denoted as DPW-ND) in this paper, to show the advantage of selecting waypoints based on sharing ability and parallel requesting for independent queries. Algorithm 4 depicts the pseudo code of DPW-ND.

In summary, DPW-ND takes advantages of Direction sharing, Parallel requesting for selected queries in $\mathcal{Q}$ based on the network distance, and Waypoints selected based on the Network Distance, while DPW-SA makes the full utilization of Direction sharing, Parallel requesting for independent queries in $\mathcal{Q}$, and Waypoints selected based on the Sharing Ability.

\subsection{Evaluation model}

\subsubsection{Performance metrics}

We evaluate the performance of the baseline approach (i.e., DPW-ND) and our proposed algorithm (i.e., DPW-SA) in terms of two metrics: (1) the average number of external route requests submitted to the Web mapping service per user query and (2) the average query response time per user query. The query response time of a query is the time from the time when the query is received by the LBS provider to the time when the answer is returned to 
the querying user; thus, it includes the local CPU processing time, the waiting time at the LBS provider, the communication time between the LBS provider and the Web mapping service, and the remote processing time at the Web mapping service provider.

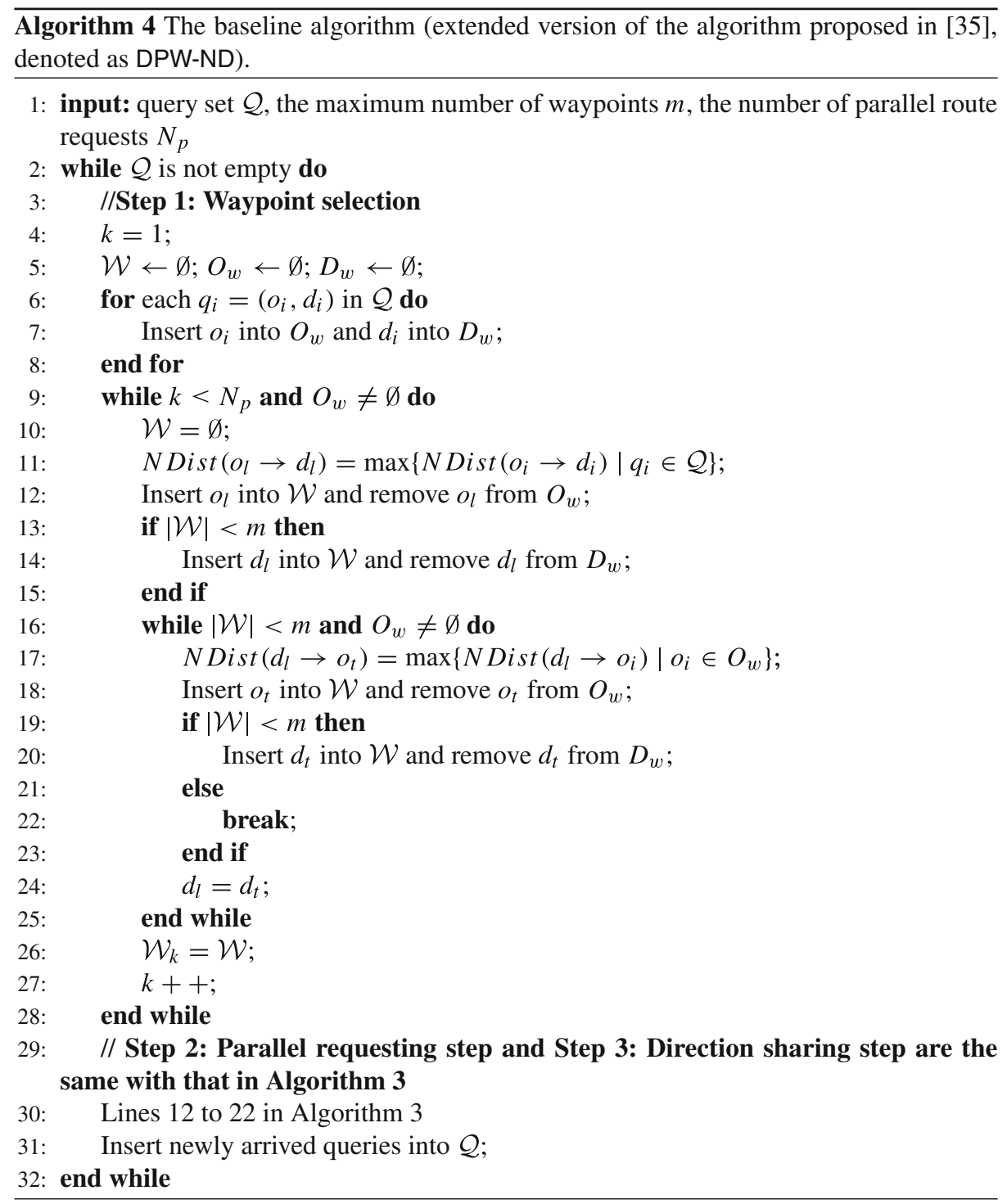

\subsubsection{Experimental Settings}

We evaluated the performance of DPW-ND and DPW-SA using Java with a real road network of Hennepin County, Minnesota, USA [30]. We selected an area of $8 \times 8 \mathrm{~km}^{2}$ that contains 6,109 road segments and 3,593 intersections, and the latitude and longitude of its leftbottom and right-top corners are (44.898441, -93.302791) and (44.970094, -93.204015), respectively. 
Unless mentioned otherwise, Bing Maps is taken as the Web mapping service in our experiments, the number of waypoints is 10 , and the number of route requests submitted to the Web mapping service in parallel is 50, i.e., $m=10, N_{p}=50$. The default number of user queries in our experiment is set to 1000 , i.e., $|\mathcal{Q}|=1000$ : the query origins are generated following Gaussian $(\sigma=3, \beta=10)$ distribution, i.e., 10 Gaussian bells are generated in the road network, the standard deviation of each Gaussian bell is set to 3, and the number of query origins in those bells follow Zipf's law, to simulate user query origins more real $[1,16]$; the query destinations are randomly selected from a real POI dataset (including 61 bars, 65 cafes, 320 restaurants, and 619 stores), which is collected from Google Places API [28] within the selected area.

\subsection{Experimental results}

We evaluate the scalability, efficiency, and applicability of the proposed algorithm (i.e., DPW-SA) and the baseline approach (i.e., DPW-ND) with respect to various numbers of queries, waypoints and parallel route requests, as well as different query distributions.

\subsubsection{Effect of the number of queries}

In this section, the number of user queries varies from 400 to 2,000 to evaluate the performance of DPW-ND and DPW-SA with respect to various numbers of queries as depicted in Fig. 5.

Without any optimization, the LBS provider needs to issue one external route request for each query. With the utilization of waypoints in a route request, only 0.2 route request per user query is needed if the number of waypoints is 10 . With the optimization of waypoints and direction sharing, DPW-ND can reduce the average number of route requests by at least $20 \%$, i.e., less than 0.16 route request per user query is needed; while, DPW-SA can further reduce it by nearly $22 \%$ on average compared with DPW-ND, which benefits from selecting waypoints based on sharing ability and parallel requesting for independent queries (Fig. 5a).

When the number of queries gets larger, there are more outstanding queries in the road network; hence, the direction information of a route has a higher chance to be shared with more queries (i.e., the power of the direction sharing optimization increases); thus, the number of external route request gets lower for both evaluated algorithms, as show in Fig. 5a. The results also show the scalability of DPW-SA with the increase of the number of queries.

As the default number of parallel route requests submitted to the Web mapping service is fixed, i.e., $N_{p}=50$, when there are more queries arrived, a query encounters a longer waiting time. Therefore, the average query response time gets longer with the increase of the

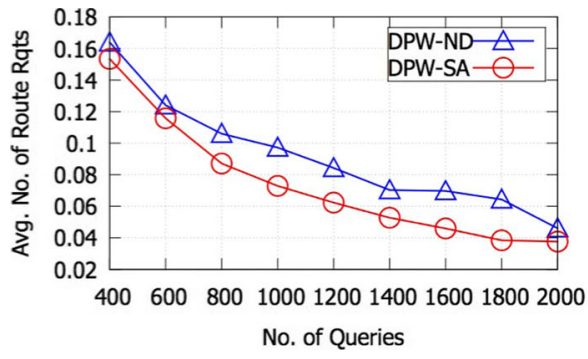

(a) Average number of external route requests

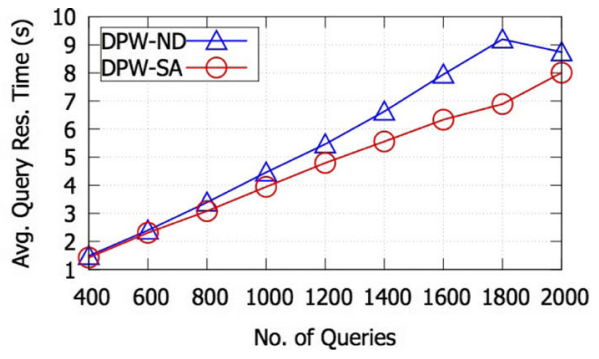

(b) Average query response time

Fig. 5 Effect of the number of queries 
number of queries, as depicted in Fig. 5b. DPW-SA yields the less average query response time compared with the baseline algorithm DPW-ND, i.e., $12 \%$ reduced.

\subsubsection{Effect of the number of waypoints in an external route request}

In this section, the number of waypoints provided by the Web mapping service provider varies from 2 to 20, to study the performance of DPW-ND and DPW-SA with respect to various numbers of waypoints, as depicted in Fig. 6.

With more waypoints utilized in an external route request, its direction information can be used to answer more queries. Therefore, when the number of waypoints gets larger, the average number of external route requests for DPW-ND and DPW-SA drops significantly (Fig. 6a). Figure 6a also shows that DPW-SA is more effective with different numbers of waypoints as it consistently yields less external requests than DPW-ND. Similarly, the average query response time of DPW-ND and DPW-SA drops gradually along with the increase of the number of waypoints (Fig. 6b). As a result, our DPW-SA can achieve not only the best number of external route requests (20\% reduced on average) but also the shortest query response time (13\% reduced on average) compared with DPW-ND.

\subsubsection{Effect of the number of parallel route requests submitted to the web mapping service}

In this section, the number of parallel route requests submitted to the Web mapping service varies from 10 to 100 , to study the performance of DPW-ND and DPW-SA with respect to various numbers of parallel route requests, as depicted in Fig. 7.

The number of external route requests of DPW-ND and DPW-SA increases when the number of parallel requests gets higher (Fig. 7a). This is because they issue more parallel route requests for queries in $\mathcal{Q}$ that will weaken the sharing power of the direction sharing optimization. It is expected that when the system is able to send more parallel route requests, it achieves a better query response time (Fig. 7b). Still, DPW-SA performs much better than DPW-ND in terms of the number of external route requests ( $23 \%$ reduced on average) and the query response time (14\% reduced on average).

\subsubsection{Effect of different query distribution}

In this section, we conduct two sets of experiment to study the performance of DPW-ND and DPW-SA with respect to different query distribution. For each query distribution $G(\sigma, \beta)$,

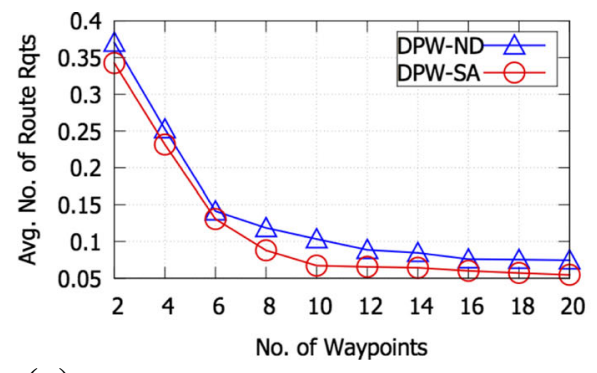

(a) Average number of external route requests

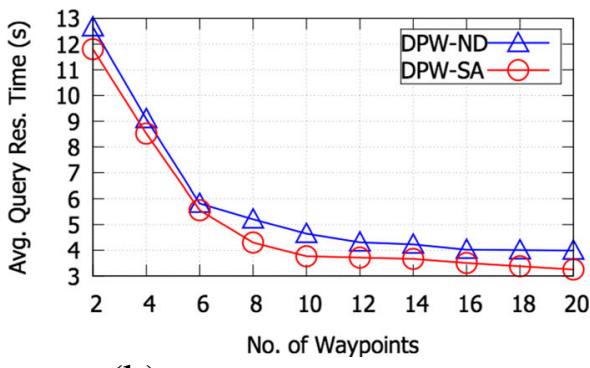

(b) Average query response time

Fig. 6 Effect of the number of waypoints in an external route request 


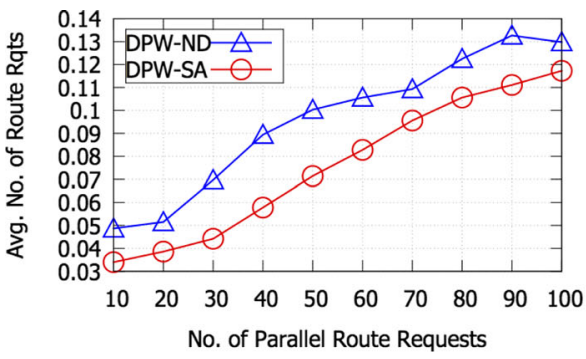

(a) Average number of external route requests

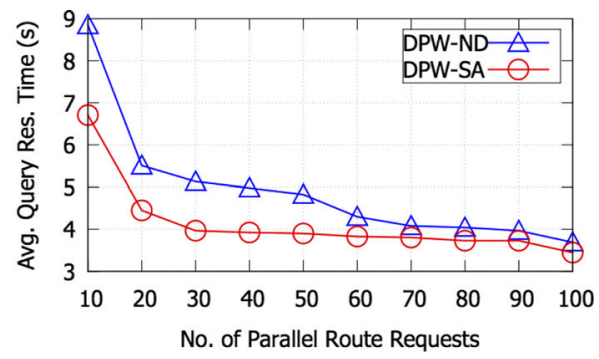

(b) Average query response time

Fig. 7 Effect of the number of parallel route requests

we select $\beta$ Gaussian bells in the road network randomly, set the standard deviation of each Gaussian bell to $\sigma$, and generate 1,000 query origins in those bells follow Zipf's law [1], while the query destinations are randomly selected from a real POI dataset.

For one set of experiment, the number of Gaussian bells varies from 1 to 20, and the standard deviation of each Gaussian bell is set to 3, as shown in Fig. 8. When the number of Gaussian bells increases, queries get more and more disperse in the road network, which weakens the sharing power of the direction sharing optimization; thus, the number of external route requests of DPW-ND and DPW-SA increases, resulting in the increase of the query response time accordingly.

For the other set of experiment, the number of Gaussian bells is set to 10, and the standard deviation of each Gaussian bell varies from 1 to 10, as shown in Fig. 9. Besides, we also generate query origins uniformly for comparison. When the standard deviation gets smaller, i.e., queries are more near to each other, queries selected by DPW-ND for parallel requesting are more 'dependent' with each other as DPW-ND selects them only based on the network distance, which leads to more unnecessary route requests are issued; therefore, both the number of external route requests and the query response time of DPW-ND is the highest when the standard deviation is the smallest. Such situation will not happen to DPW-SA, since it selects queries for parallel requesting based on the sharing ability, i.e., independent queries are selected.

Observe that no matter any Gaussian distribution or Uniform distribution, our DPW-SA outperforms DPW-ND on both the number of external route requests (26\% reduced on average) and the query response time ( $15 \%$ reduced on average).

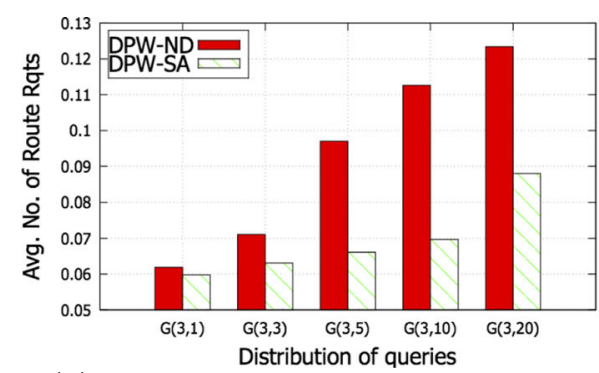

(a) Average number of external route requests

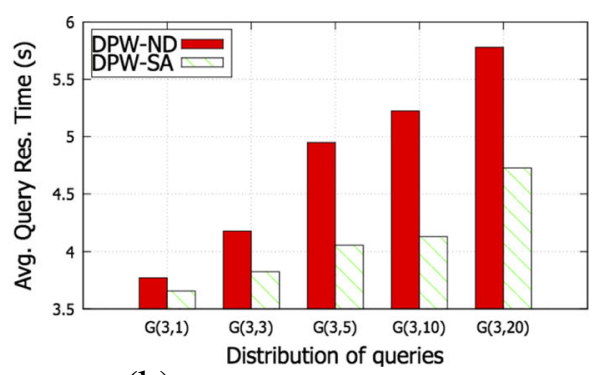

(b) Average query response time

Fig. 8 Effect of different query distribution (with different number of Gaussian bells) 


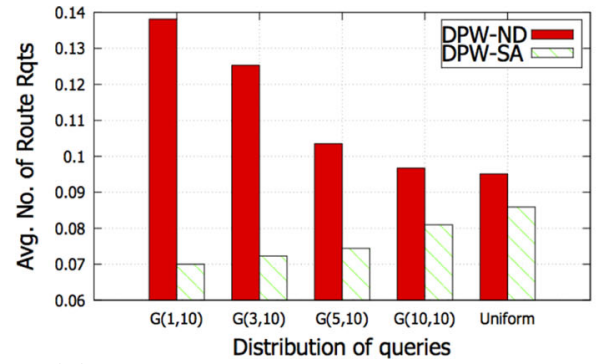

(a) Average number of external route requests

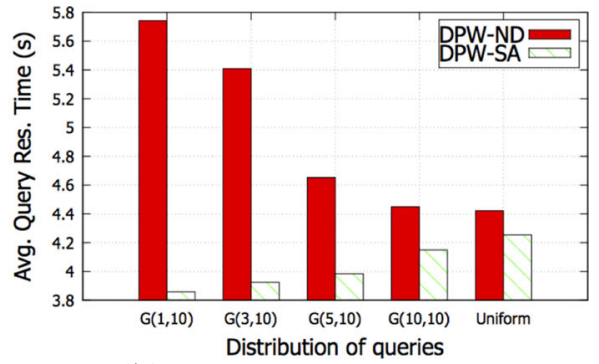

(b) Average query response time

Fig. 9 Effect of different query distribution (with different standard deviation)

\section{Conclusion}

In this paper, we have proposed a spatial mashup framework for a location-based service (LBS) provider to retrieve travel route information between two location points in a road network through issuing an external route request to a Web mapping service, e.g., Google Maps, Bing Maps, and MapQuest Maps. Since retrieving external data is much more expensive than accessing local data, we employ the direction sharing, parallel requesting and route waypoint optimizations to reduce the number of such external route requests and the query response time for users. To reflect the possibility of sharing the direction information of a route, we first formally define a method to measure its sharing ability, and find out the queries that their querying routes are independent with each other for parallel processing. Then, we model the problem of selecting the optimal set of waypoints in a road network for an external route request, i.e., the sharing ability of the sub-routes in a route request should be maximized, as the problem of finding the longest simple path in a weighted complete digraph. As it is a MAX SNP-hard problem, i.e., there is no polynomial-time algorithm that can even find a constant factor approximation for the problem unless $\mathrm{P}=\mathrm{NP}$, we propose a greedy algorithm with performance guarantee to find the best set of waypoints in an external route request. We evaluate the performance of our algorithm using a real Web mapping service, a real road network, real and synthetic data sets. Our experimental results show that our proposed algorithm is efficient and scalable with various numbers of queries, waypoints and parallel route requests, as well as different query distributions.

Acknowledgments Research reported in this publication was partially supported by King Abdullah University of Science and Technology (KAUST), the Fundamental Research Funds for the Central Universities in China (Project No. JUSRP11557), the National Natural Science Foundation of China (Project No. 61572336 and 61472337), and a Strategic Research Grant from City University of Hong Kong (Project No. 7004420).

\section{References}

1. Bruno N, Chaudhuri S, Gravano L (2001) Stholes: a multidimensional workload-aware histogram. In: ACM SIGMOD

2. Bruno N, Gravano L, Marian A (2002) Evaluating top- $k$ queries over web-accessible databases. In: IEEE ICDE

3. Chang KCC, Hwang SW (2002) Minimal probing: supporting expensive predicates for top- $k$ queries. In: ACM SIGMOD

4. Cormen TH, Leiserson CE, Rivest RL, Stein C (2009) Introduction to algorithms, 3rd edn. MIT Press 
5. Demiryurek U, Banaei-Kashani F, Shahabi C, Ranganathan A (2011) Online computation of fastest path in time-dependent spatial networks. In: SSTD

6. Ding B, Yu JX, Qin L (2008) Finding time-dependent shortest paths over large graphs. In: EDBT

7. George B, Kim S, Shekhar S (2007) Spatio-temporal network databases and routing algorithms: a summary of results. In: SSTD

8. Google Maps: http://maps.google.com

9. Google Maps/Google Earth APIs Terms of Service: http://code.google.com/apis/maps/terms.html

10. Gutin G, Punnen AP (2006) The traveling salesman problem and its variations. Springer Science \& Business Media

11. Huang X, Cheng H, Li RH, Qin L, Yu JX (2013) Top-k structural diversity search in large networks. VLDB J 6(13):1618-1629

12. INRIX: http://www.inrix.com

13. Kosaraju SR, Park JK, Stein C (1994) Long tours and short superstrings. In: FOCS

14. Levandoski JJ, Mokbel MF, Khalefa ME (2010) Preference query evaluation over expensive attributes. In: CIKM

15. Li J, Liu C, Liu B, Mao R, Wang Y, Chen S, Yang JJ, Pan H, Wang Q (2015) Diversity-aware retrieval of medical records. Comput Ind 69:81-91

16. Li Y, Yiu ML (2015) Route-saver: leveraging route apis for accurate and efficient query processing at location-based services. IEEE TKDE 27(1):235-249

17. Liu A, Zheng K, Li L, Liu G, Zhao L, Zhou X (2015) Efficient secure similarity computation on encrypted trajectory data. In: IEEE ICDE, pp 66-77

18. Lu M, Tang Y, Sun R, Wang T, Chen S, Mao R (2015) A real time displacement estimation algorithm for ultrasound elastography. Comput Ind 69:61-71

19. Mao R, Xu H, Wu W, Li J, Li Y, Lu M (2015) Overcoming the challenge of variety: big data abstraction, the next evolution of data management for aal communication systems. IEEE Commun Mag 53(1):42-47

20. Mao R, Zhang P, Li X, Liu X, Lu M (2016) Pivot selection for metric-space indexing. Int J Mach Learn Cybern 7(2):311-323

21. MapQuest Directions Web Service: http://www.mapquestapi.com/directions

22. MapQuest Maps: http://www.mapquestapi.com

23. Microsoft Bing Maps: http://www.bing.com/maps

24. Navteq: https://www.navteq.com/

25. ProgrammableWeb: http://www.programmableweb.com/category-api

26. Sommer C (2014) Shortest-path queries in static networks. ACM Comput Surv, CSUR 46(4):45

27. The Google Directions API: https://developers.google.com/maps/documentation/directions

28. The Google Places API: https://developers.google.com/places/

29. Thomsen JR, Yiu ML, Jensen CS (2012) Effective caching of shortest paths for location-based services. In: ACM SIGMOD

30. TIGER/Line Shapefiles 2009 for: Hennepin County, Minnesota: http://www2.census.gov/cgi-bin/ shapefiles2009/county-files?county=27053

31. U LH, Zhao HJ, Yiu ML, Li Y, Gong Z (2014) Towards online shortest path computation. IEEE TKDE 26(4):1012-1025

32. Wang J, Huang JZ, Guo J, Lan Y (2015) Recommending high-utility search engine queries via a queryrecommending model. Neurocomputing 167:195-208

33. Wu L, Xiao X, Deng D, Cong G, Zhu AD, Zhou S (2012) Shortest path and distance queries on road networks: an experimental evaluation. In: VLDB

34. Yelp, Inc.: http://www.yelp.com

35. Zhang D, Chow CY, Li Q, Liu A (2016) Efficient evaluation of shortest travel-time path queries in road networks by optimizing waypoints in route requests through spatial mashups. In: APWEb

36. Zhang D, Chow CY, Li Q, Zhang X, Xu Y (2011) Efficient evaluation of k-NN queries using spatial mashups. In: SSTD

37. Zhang D, Chow CY, Li Q, Zhang X, Xu Y (2013) SMAshq: Spatial mashup framework for k-NN queries in time-dependent road networks. Distributed and Parallel Databases, DAPD 31(2):259-287

38. Zhang D, Chow CY, Li Q, Zhang X, Xu Y (2016) A spatial mashup service for efficient evaluation of concurrent k-nn queries. IEEE Trans Comput 65(8):2428-2442

39. Zhu AD, Ma H, Xiao X, Luo S, Tang Y, Zhou S (2013) Shortest path and distance queries on road networks: towards bridging theory and practice. In: ACM SIGMOD

40. Zhu CJ, Lam KY, Cheng RC, Poon CK (2015) On using broadcast index for efficient execution of shortest path continuous queries. Inf Syst 49:142-162

41. Zhu Z, Xiao J, Li J, Wang F, Zhang Q (2015) Global path planning of wheeled robots using multiobjective memetic algorithms. Integrated Computer-Aided Engineering 22(4):387-404 


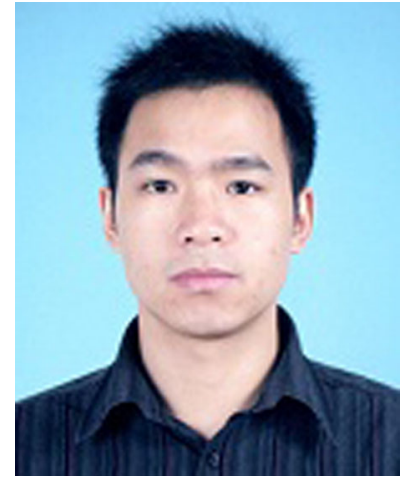

Detian Zhang received the joint $\mathrm{PhD}$ degree in computer science from the University of Science and Technology of China and City University of Hong Kong in 2014. He is currently a lecturer with the School of Digital Media, Jiangnan University, Wuxi, China. His research interests include location-based services, spatio-temporal databases, and wireless networks.

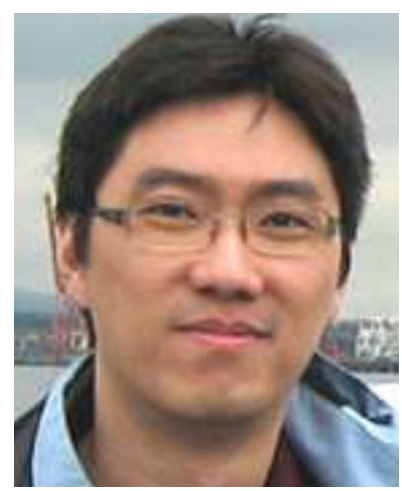

Chi-Yin Chow received B.A. and MPhil degrees in computing science from The Hong Kong Polytechnic University in 2002 and 2005, respectively, and the M.S. and Ph.D. degrees from the University of MinnesotaTwin Cities in 2008 and 2010, respectively. He is currently an assistant professor in Department of Computer Science, City University of Hong Kong. His research interests include data analytics, spatial and spatiotemporal databases, GIS, data privacy, and mobile computing. He is the co-founder and co-organizer of ACM SIGSPATIAL MobiGIS 2012, 2013, and 2014. 


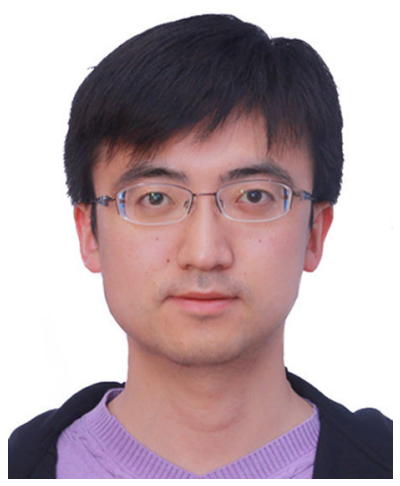

An Liu received his Ph. D. degree in computer science from both City University of Hong Kong and University of Science and Technology of China in 2009. He is currently a postdoctoral fellow at King Abdullah University of Science and Technology (KAUST). His research interests include spatial databases, data mining, data security and privacy. He has published more than 70 papers in referred journals and conferences, including TKDE, TSC, ICDE, WWW etc.

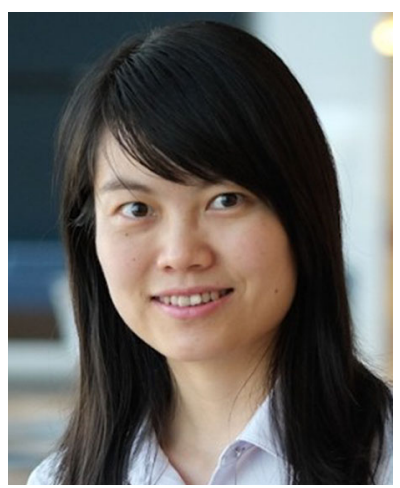

Xiangliang Zhang is an Assistant Professor and directs the Machine Intelligence and kNowledge Engineering (MINE) Laboratory in King Abdullah University of Science and Technology (KAUST). She earned her Ph.D. degree in computer science from INRIA-University Paris-Sud 11, France, in July 2010. Her main research interests and experiences are in diverse areas of machine learning and data mining. She has published over 70 papers in referred journals and conferences, including TKDE, SIGKDD, VLDB J, AAAI, IJCAI, ICDM, ECML/PKDD, CIKM, InfoCom etc. 


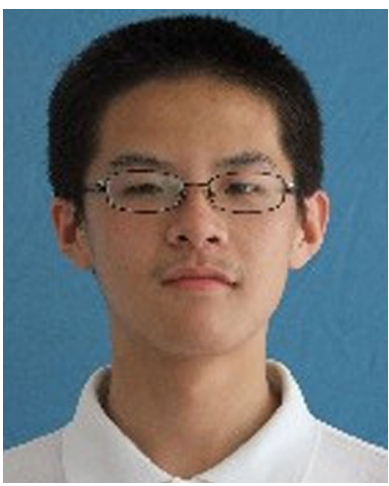

Qingzhu Ding is an undergraduate in the Department of Computer Science \& Technology at Soochow University. His main research interests include database and data mining.

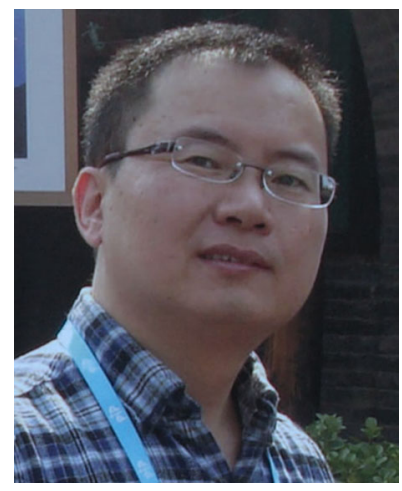

Qing Li received the B.Eng. degree from Hunan University, Changsha, China, and the M.Sc. and Ph.D. degrees from the University of Southern California, Los Angeles, all in computer science. He is currently a Professor with the Department of Computer Science, City University of Hong Kong, Kowloon, Hong Kong. His current research interests include dynamic object modeling, multimedia and mobile information retrieval and management, distributed databases and data warehousing/mining, and workflow management and web services. 